\title{
ARTICLE Rislenemdaz treatment in the lateral habenula improves despair-like behavior in mice
}

\author{
Ting Lei ${ }^{1,2}$, Dan Dong ${ }^{3}$, Meiying Song ${ }^{1}$, Yanfei Sun ${ }^{2}$, Xiaofeng Liu' ${ }^{1}$ and Hua Zhao ${ }^{1,2}$
}

The specific GluN2B antagonist rislenemdaz (Ris; a.k.a. MK-0657 and CERC-301) is in phase II clinical trial as an antidepressive drug, but the working mechanism for its antidepressant effects is not clearly understood. Given the important role of the lateral habenula $(\mathrm{LHb})$ in the pathogenesis of depression and the fact that GluN2B-containing N-methyl-D-aspartate receptors and brain-derived neurotrophic factor (BDNF) are expressed in the LHb, we conducted a study to examine whether the LHb mediates Ris' antidepressant effects in a chronic restraint stress (CRS)-induced depressive-like mouse model. In this study, Ris was administered systemically or locally into the LHb. Short hairpin RNAs were used to knockdown BDNF in the LHb. Depressive-like behaviors were assessed with the open field test, forced swimming test, tail suspension test, and sucrose preference test. Expression of GluN2B, BDNF, and c-Fos in the LHb were analyzed with western blotting and immunohistochemistry under condition with Ris administered systemically or with BDNF knockdown in the LHb. We found that both systemic and intra-LHb administration of Ris alleviated CRSinduced despair-like behavior and that systemic Ris reduced LHb expression of GluN2B, BDNF, and c-Fos (a neuronal activity marker). Specific knockdown of BDNF in the LHb prevented CRS-induced despair-like behavior, while preventing CRS-induced increases in BDNF and c-Fos expression in the LHb. Together these results suggest that Ris may exert its antidepressant effects through affecting the LHb such as downregulating BDNF expression in the LHb.

Neuropsychopharmacology (2020) 45:1717-1724; https://doi.org/10.1038/s41386-020-0652-9

\section{INTRODUCTION}

Major depressive disorder (MDD) is a common neuropsychiatric disorder that afflicts more than 300 million people of all ages [1] and it imposes a heavy economic burden on society and families. Given the fact that almost a third of patients with MDD are resistant to treatment with medication and psychotherapy [2], it is important to better understand the pathophysiology of MDD to help develop more effective therapies.

Many studies have shown that glutamatergic neurotransmission is closely associated with the pathophysiology and treatment responsivity of MDD [3, 4]. Elevated glutamate levels and decreased glutamine/glutamate ratios, amenable to normalization with antidepressant drugs, have been reported in plasma samples obtained from patients with MDD [5]. Recently, the N-methyl-Daspartate receptor (NMDAR), an ionotropic glutamate receptor, has been identified to be a therapeutic target for the treatment of depression [6, 7]. In preclinical studies, NMDAR antagonism has been shown to produce rapid and sustained antidepressant effects in both rodent models and human subjects [8-10]. However, the use of many NMDAR antagonists, such as MK-801 and ketamine, are limited due to their harmful psychosis-like side-effects [11].

NMDARs are large heterotetrameric membrane protein complexes, most of which are composed of two obligatory GluN1 and two GluN2 subunits, the latter of which occurs in four subtypes, i.e., GluN2A, B, C, and D [12, 13]. Both genetic and pharmacological studies have suggested that GluN2B-containing NMDARs play an important role in mood disorders [14]. Specific knockout of
GluN2B in cortical pyramidal neurons has been reported to alleviate depressive-like behaviors and occlude further antidepressant action of ketamine [15]. Selective GluN2B subunit blockers also produce rapid antidepressant effects in both rodents $[16]$ and humans $[17,18]$, with low neurotoxicity and mild or negligible psychogenic effects [19]. One such antagonist that is highly selective for GluN2B-containing NMDARs, rislenemdaz (Ris), has been submitted to phase II antidepressant clinical studies. However, the mechanism mediating the antidepressant effects of this drug has not yet been clarified.

GluN2B-containing NMDARs are widely expressed in the central nervous system and have multiple physiological functions, such as regulating neuronal development [20] and synaptic plasticity [21]. Many studies have demonstrated that neurotrophins, a family of growth factor hormones, including nerve growth factor (NGF), brain-derived neurotrophic factor (BDNF), neurotrophin 4 (NT4), and neurotrophin 3 (NT3), regulate neurite growth and synaptic plasticity, and thus have been implicated in the pathophysiological processes underlying depression, especially BDNF $[22,23]$. Studies indicate that BDNF expression in the medial prefrontal cortex (mPFC) [24] and hippocampus [25] can be regulated by GluN2B-containing NMDAR activation suggesting that depression-like behaviors might be improved by BDNF regulation by way of antagonism of GluN2B-containing NMDARs, such as with Ris.

GluN2B-containing NMDARs are expressed in the lateral habenula (LHb) as evidenced by immunohistochemistry [26]. Meanwhile, abnormal activity in the LHb is strongly correlated

\footnotetext{
${ }^{1}$ Neuroscience Research Center, First Hospital of Jilin University, Changchun 130021, PR China; ${ }^{2}$ Department of Physiology, College of Basic Medical Sciences, Jilin University, Changchun 130021, PR China and ${ }^{3}$ Department of Nephrology, First Hospital of Jilin University, Changchun 130021, PR China Correspondence: Hua Zhao (zhua@jlu.edu.cn)
} 
with MDD pathophysiology [27]. Glucose metabolism in the LHb is elevated in rodent models of depression (induced by stress, injections of a-methylpara-tyrosine, or withdrawal from chronic amphetamine) [28], and electrolytic lesions of the LHb can reduce depressive-like behaviors via increasing serotonin levels in the dorsal raphe nucleus [29]. Moreover, blockade of LHb NMDARs alleviates depression-related behaviors in rats [30]. In depressive patients, deep brain stimulation of the LHb has been reported to produce rapid, sustained relief from treatment-resistant MDD [31]. However, the mechanism regarding how GluN2B-containing NMDARs in the LHb impact depression is unknown.

Based on the study findings mentioned, we hypothesized that Ris may mediate its antidepressant effects via GluN2B-containing NMDARs and BDNF in the LHb. To test our hypothesis, in the current study we determined whether Ris treatment could improve signs of behavioral despair and, if so, whether these effects involved the LHb. Specifically, we examined the effects of systemic Ris on GluN2B, BDNF, and c-Fos (neuronal activity marker) expression in the LHb. We then tested whether specific depletion of BDNF in the $\mathrm{LHb}$ could prevent chronic restraint stress (CRS)-induced despair-like behavior and CRS-induced enhancement of LHb neuronal activity.

\section{MATERIALS AND METHODS}

\section{Animals}

Male C57BL/6J mice (7 weeks old, $18-22 \mathrm{~g}$ ) were purchased from the Department of Experimental Animals at Jilin University, Changchun, China. Pairs of mice were housed in isolation ventilation cages under standard conditions $\left(22 \pm 1^{\circ} \mathrm{C}, 12 \mathrm{~h}\right.$ light/dark cycle, lights on at 7:00 a.m.) and allowed free access to food and water. The animals were housed singly for $\sim 1.5$ days before being administered the drug treatment and behavioral testing. All experiments in this study were approved by the Committee for Animal Care Research at Jilin University. Numbers and groups of mice used for different experiments are provided in the corresponding figure legends. All mice with incorrect LHb injection sites were excluded. Data presented in the same figure were obtained from the same batch of mice.

CRS

The CRS procedure was implemented as described in detail previously [32]. Briefly, mice were restrained individually in a well-ventilated 50 $\mathrm{ml}$ conical tube for $\sim 2.5 \mathrm{~h}$ per day for 14 consecutive days, and control mice remained in their home cages. After each day of CRS, restrained mice were immediately returned to their home cages.

\section{Drug administration}

Systemic Ris injection. Ris (MedChemExpress, Shanghai, China; catalog no. HY-106441A) is a highly selective GluN2B antagonist; its chemical name is 4-methylbenzyl (3S, 4R)-3-fluoro-4-[(pyrimidin-2ylamino) methyl] piperidine-1-carboxylate. Ris was first dissolved in dimethyl sulfoxide (Solarbio, Beijing, China) to make a stock solution at a concentration of $0.01 \mathrm{mg} / \mu \mathrm{l}$. Injection solutions of Ris were prepared by diluting its stock solution in saline solution with $20 \%$ sulfobutyl ether- $\beta$-cyclodextrin (Yuanye, Shanghai, China) and the amount of injection for each mouse was calculated based on the group dose level and individual body weight. The mice were administered an intra-peritoneal (i.p.) injection of Ris $(0.1,0.3,0.6$, or $1.0 \mathrm{mg} / \mathrm{kg}$ ) or vehicle twice before behavioral testing, $24 \mathrm{~h}$ and $45 \mathrm{~min}$ before the test as described previously [33].

Cannula implantation and local administration of Ris. Mice were anesthetized with an i.p. injection of $10 \%$ chloral hydrate $(0.35 \mathrm{~g} / \mathrm{kg})$ and their heads were fixed in a stereotaxic apparatus (RWD Life Science, Shenzhen, China). Lidocaine was used to numb the scalp skin before surgery. To avoid damaging large blood vessels, the guide cannulas (outer diameter, $0.41 \mathrm{~mm}$; inner diameter, $0.25 \mathrm{~mm}$ ) were placed at a $20^{\circ}$ angle relative to the midline and implanted onto the surface of LHb (coordinates relative to bregma: posterior, $-1.60 \mathrm{~mm}$; lateral, $\pm 1.25 \mathrm{~mm}$; and ventral, -2.3 to $-2.4 \mathrm{~mm}$ ) and affixed to the skull with jeweler's screws and dental cement. A dummy cannula (diameter, $0.20 \mathrm{~mm}$ ) that extended $0.2 \mathrm{~mm}$ beyond the bottom of the guide cannula was inserted into each guide cannula. After surgery, mice were placed immediately onto a heating pad until they awoke. After a 7-days recovery period, Ris or vehicle was administrated into the LHb bilaterally ( $4 \mathrm{nmol}$ in $0.2 \mu$ l each side) via corresponding injector needles (outer diameter, $0.21 \mathrm{~mm}$; inner diameter, $0.11 \mathrm{~mm}$ ) that were attached to an automated syringe pump (Longer Precision Pump Co., Ltd, Baoding, China). The injector needles were left in place for $5 \mathrm{~min}$ to ensure that the drug diffused into the brain tissue completely. Behavioral testing was conducted 30 min after the drug diffusion period.

Knockdown of BDNF in the $\mathrm{LHb}$

Short hairpin RNA (shRNA) against mouse BDNF (5'-GAAGTAA ACGTCCACGGACAA-3') and scrambled shRNA (5'-CGCTGAGTACT TCGAAATGTC- $3^{\prime}$ ) were each inserted into an adeno-associated virus (AAV) vector containing the enhanced green fluorescence protein coding sequence (Genechem, Shanghai, China) to form AVV-shBDNF and AVV-scramble, respectively. AVV-shBDNF or AVVscramble was injected vertically into the $\mathrm{LHb}$ of anesthetized mice $\left(1 \times 10^{12}\right.$ viral particles per $\mathrm{ml}, 0.15 \mu \mathrm{l}$ each side; coordinates relative to bregma: posterior, $-1.60 \mathrm{~mm}$; lateral $\pm 0.4 \mathrm{~mm}$; ventral, -2.5 to $-2.7 \mathrm{~mm}$ ). After injection, the needle was withdrawn slowly and the incision was sutured.

\section{Behavioral testing}

Open field test (OFT). Mice were placed in the central area $(20 \mathrm{~cm} \times$ $20 \mathrm{~cm}$ ) of a cubic arena $(50 \mathrm{~cm} \times 50 \mathrm{~cm} \times 50 \mathrm{~cm})$, which they were allowed to explore freely for $20 \mathrm{~min}$. Between tests, the open-field apparatus was cleaned with $75 \%$ alcohol to remove the smell of the last mouse. The activity of each mice was tracked and analyzed by an EthoVision XT 8.5 system (Noldus, Wageningen, Netherlands).

Forced swimming test (FST). Mice were placed individually into a Plexiglas cylinder (inner diameter, $11 \mathrm{~cm} ; 30 \mathrm{~cm}$ high) containing water ( $24 \pm 1{ }^{\circ} \mathrm{C} ; 10 \mathrm{~cm}$ deep) and allowed to swim freely for $6 \mathrm{~min}$. After each test, the cylinder was cleaned and refilled with fresh water. Immobility time during the last 4 min of the 6 min tests was measured.

Tail suspension test (TST). Mice were suspended $5 \mathrm{~cm}$ above the horizontal floor for 6 min by fixing the distal $1 / 3$ of the tail with adhesive tapes. Immobility time during the last 4 min of the 6 min TST period was measured. Mice were considered immobile only when their body and four limbs were still.

Sucrose preference test (SPT). Each mouse was habituated to the test for 2 days by exposure to two bottles of water, then exposed to two $2 \%$ sucrose solution bottles for 2 days. Subsequently, the mice were deprived of water and sucrose solution for $24 \mathrm{~h}$. Finally, the mice were tested with a water bottle and a $2 \%$ sucrose solution bottle for $2 \mathrm{~h}$. The positions of the water and sucrose bottles were switched halfway through the test. Mean sucrose consumption ratio, relative to total consumption, was determined for the first and second hour of the test to detect preference [34].

\section{Immunohistochemistry}

Each mouse was anesthetized with an injection of $10 \%$ chloral hydrate $(0.35 \mathrm{~g} / \mathrm{kg}$, i.p.), and its brain was extracted quickly and soaked in $10 \%$ neutral formalin solution for $24 \mathrm{~h}$. The brains were dehydrated, embedded in paraffin, and cut into $4 \mu \mathrm{m}$ sections on a Leica microtome (RM2245). The sections were de-paraffinized with xylene. Then brain tissues were rehydrated by placing them in pure ethanol followed by a series of ethanol solutions $(90,80,70$, and $50 \%$ ) diluted with distilled water. After a 5 min recovery period in 
citrate at $95^{\circ} \mathrm{C}$, the sections were washed three times ( $5 \mathrm{~min} /$ wash) with phosphate buffered saline.

An MXB immunohistochemical kit was used according to the manufacturer's suggested protocol (Fuzhou, China; catalog no. KIT9710). Blocking reagent (reagent $A$ ), goat serum (reagent $B$ ), rabbit anti-c-Fos antibody (1:400; Abcam, Cambridge, UK; catalog no. ab190289), biotin-labeled anti-rabbit lgG second antibody (reagent $C)$, and horseradish peroxidase (HRP)-labeled streptomycete antibiotin protein (reagent $D$ ) were added in sequence $(A, B$, primary antibody, $C$, and then D). After washing, the sections were placed in a color developing agent (MXB, Fuzhou, China; catalog no. DAB0031) and the color reaction was terminated with water when the color reached an appropriate saturation. Nuclei were counterstained with Mayer's hematoxylin solution. The sections were examined under a light microscope (Olympus IX71) and c-Fos-immunopositive neurons were counted as an index of neuronal excitability.

Western blot

The $\mathrm{LHb}$ regions were dissected out of each brain and proteins were extracted from the $\mathrm{LHb}$ with a protein extraction kit
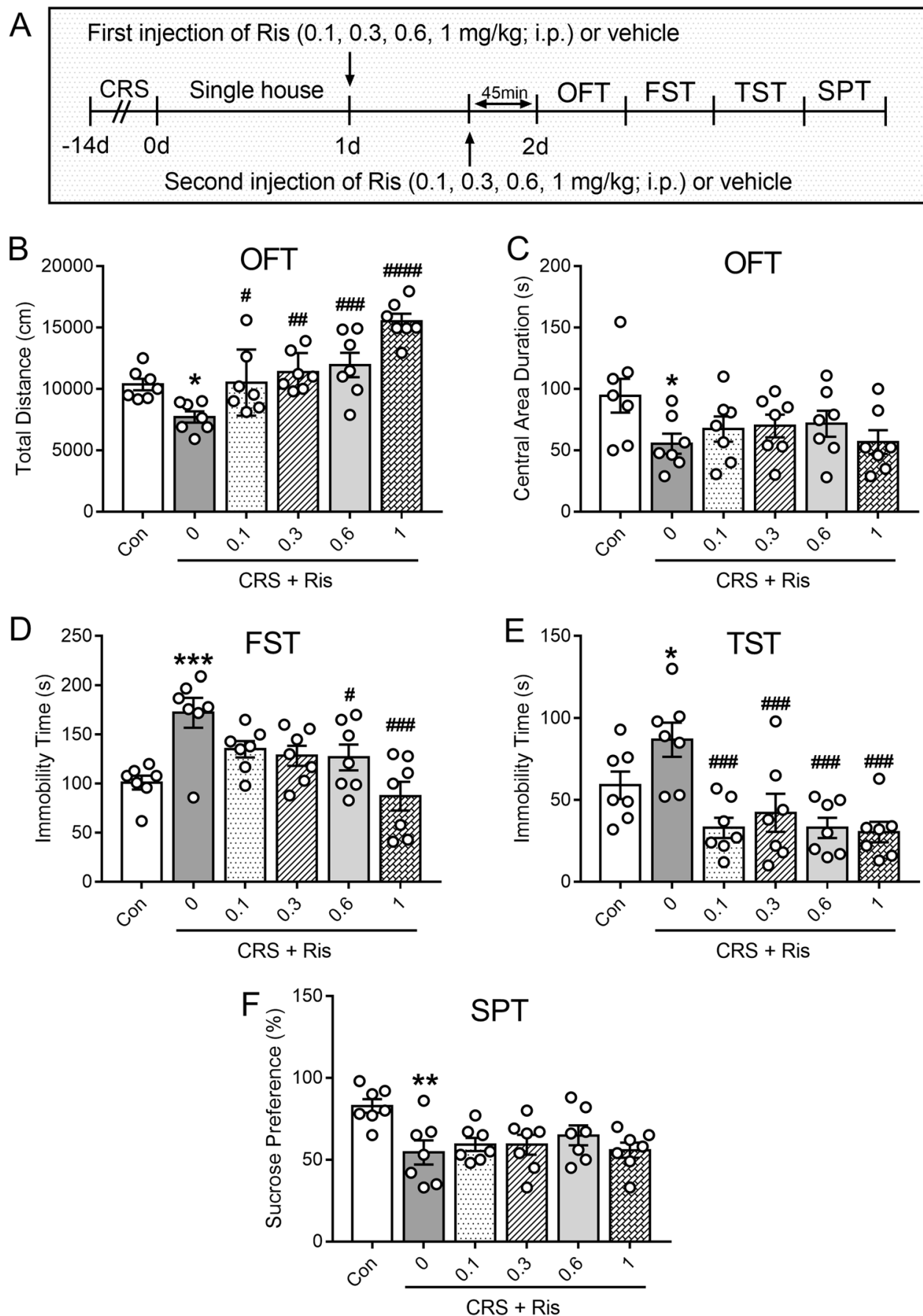

Fig. 1 Systemic Ris alleviates CRS-induced depressive-like behaviors in mice. Mice subjected to CRS were given Ris (in mg/kg, i.p.: 0.1, 0.3, 0.6 , or 1.0; CRS + Ris groups) or vehicle (CRS + Vehicle group). Normal mice not subjected to CRS were given vehicle (Con group). a Timeline of Ris administration and behavioral testing, $n=7 /$ group. b Total distance traveled in the OFT. $\mathbf{c}$ Central area duration in the OFT. d Immobility time in the FST. e Immobility time in the TST. f Sucrose preference in the SPT. Data are means \pm SEMs; ${ }^{*} p<0.05 ;{ }^{* *} p<0.01 ;{ }^{* * *} p<0.001$ vs. Con

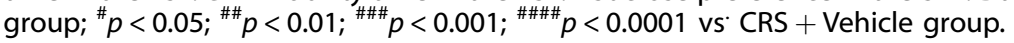


(Solarbio, Beijing, China). A $30 \mu \mathrm{g}$ aliquot of proteins were separated by $10 \%$ sodium dodecyl sulfate-polyacrylamide gel electrophoresis and transferred onto PVDF membranes. The membranes were blocked with $5 \%$ skim milk at room temperature for $1 \mathrm{~h}$ and then incubated with the following primary antibodies at $4{ }^{\circ} \mathrm{C}$ overnight: rabbit anti-GluN2B (1:1000; Abcam, Cambridge, UK; no. ab65783), rabbit anti-c-Fos (1:1000; Abcam; no. ab190289), mouse anti-NGF (1:500; Bioss, Beijing, China; no. bsm-10806M), mouse anti-BDNF (1:500; Bioss; no. bsm-2291M), rabbit anti-NT4 (1:500; Bioss; no. bs-0158R), rabbit anti-NT3 (1:500; Bioss; no. bs21490R), and anti-GAPDH (1:5000; Abcam; no. ab181602). After washing, membranes were incubated with HRP-conjugated goat anti-rabbit (1:5000; Bioss, Beijing, China; no. bs-0295G-HRP) or goat anti-mouse (1:5000; Bioss, Beijing, China; no. bs-40296G-HRP) secondary antibody at room temperature for $1 \mathrm{~h}$. After washing, a highly sensitive ECL reagent (Tanon, Shanghai, China) was placed on the membrane for $1 \mathrm{~min}$. Bands were detected with SageCapture software (Sage Creation Science Co., Ltd, Beijing, China) and measured with ImageJ software (NIH).

Statistical analysis

SPSS 23 and Graphpad Prism 7.0 were used to analyze data and generate graphs. Data were analyzed with Student's $t$ test (Fig. 4c, Figs. S1, S2) and one-way analyses of variance (ANOVAs; Figs. 1-4,
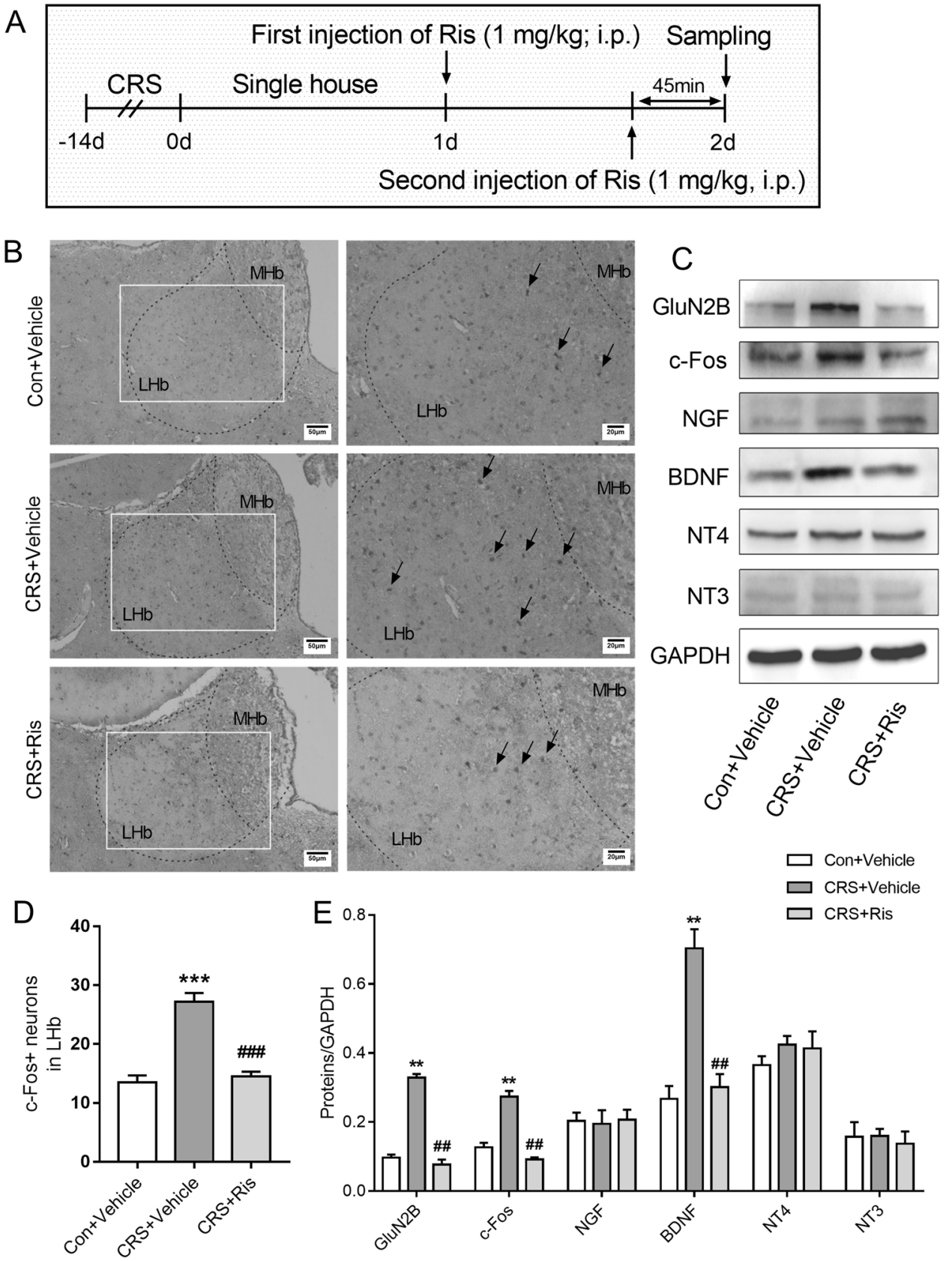

Fig. 2 Systemic Ris reverses CRS effects on GluN2B, BDNF, and c-Fos expression in the LHb. Mice subjected to CRS were given vehicle (CRS + Vehicle group) or Ris (1 mg/kg, i.p.; CRS + Ris group). Normal mice not subjected to CRS were given vehicle (Con + Vehicle group). a Timeline of Ris administration and protein detection, $n=4-6 /$ group. $\mathbf{b}$ Representative c-Fos immunolabeled sections (scale bar $=50 \mu \mathrm{m}$, left; scale bar $=20 \mu \mathrm{m}$, right). Black arrows indicate c-Fos positive cells. c Representative western blot images. d Quantitative analysis of c-Fos positive neurons in the LHb. e Quantitative analysis of western blots shown in (c). Data are means \pm SEMs; ${ }^{* *} p<0.01 ;{ }^{* * *} p<0.001$ vs. Con + Vehicle group; ${ }^{\# \#} p<0.01 ;{ }^{\# \# \#} p<0.001$ vs. CRS + Vehicle group. 


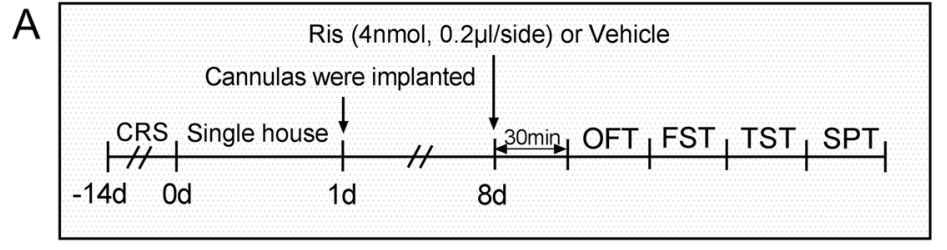

$\mathrm{B}$
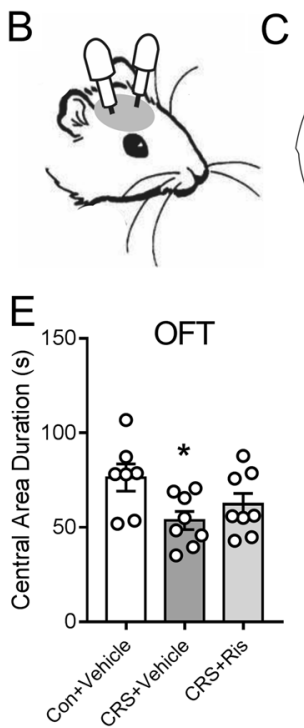

C

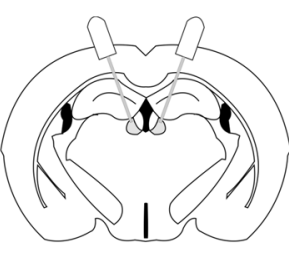

$\mathrm{F}$

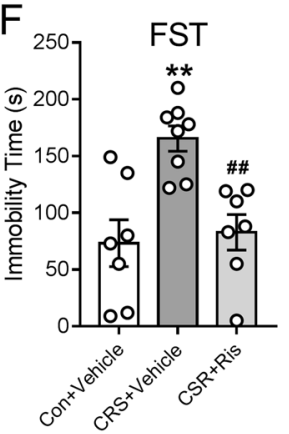

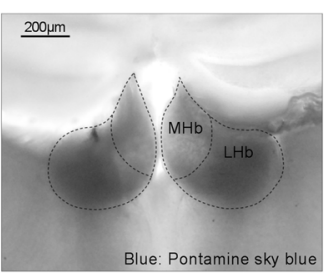

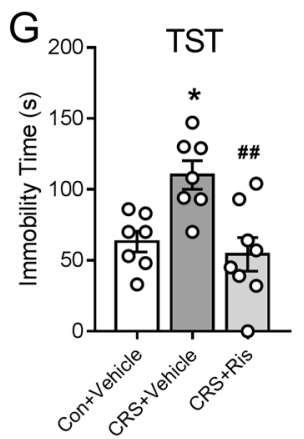

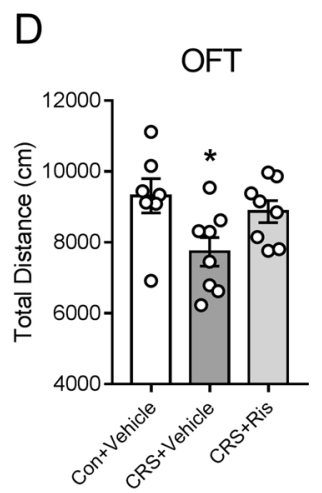

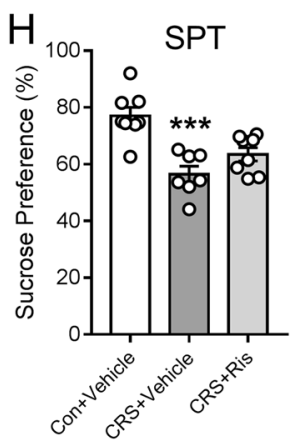

Fig. 3 Intra-LHb injection of Ris improves CRS-induced despair-like behavior. Mice subjected CRS were given intra-LHb injection of Ris (CRS + Ris group) or vehicle (CRS + Vehicle group). Behavioral control mice not subjected to CRS were given intra-LHb vehicle (Con + Vehicle group). a Timeline of Ris administration and behavioral testing, $n=7-8 /$ group. $\mathbf{b}$ Cannula implantation. $\mathbf{c}$ Map of Ris injection (left) and a coronal brain section stained with Pontamine sky blue dye to show Ris injection site (right). d Total distance traveled in the OFT. e Central area duration in the OFT. $\mathbf{f}$ Immobility time in the FST. $\mathbf{g}$ Immobility time in the TST. $\mathbf{h}$ Sucrose preference in the SPT. Data are means \pm SEMs; ${ }^{*} p<0.05 ;{ }^{* *} p<0.01 ;{ }^{* *} p<0.001$ vs. Con + Vehicle group; ${ }^{\# \#} p<0.01$ vs. CRS + Vehicle group.

except Fig. 4c). Fisher's least-significant difference test was used for post hoc testing. All data are presented as means \pm standard errors (SEMs). $P$ values $<0.05$ were considered statistically significant.

\section{RESULTS}

Systemic Ris improved depressive-like behaviors

CRS mice were injected with Ris at $0.1,0.3,0.6$, or $1.0 \mathrm{mg} / \mathrm{kg}$ (i.p.) and then subjected to behavioral testing, including the OFT, FST, TST, and SPT. Relative to the Con group, CRS-exposed mice showed augmented depressive-like behaviors, including decreased total distance $\left(F_{5,36}=12.309, p<0.05\right.$, Fig. $\left.1 \mathrm{~b}\right)$ and central area duration $\left(F_{5,36}=1.825, p<0.05\right.$, Fig. $\left.1 c\right)$ in the OFT, increased immobility time in the FST $\left(F_{5,36}=6.135, p<0.001\right.$, Fig. 1d) and TST $\left(F_{5,36}=6.736, p<0.05\right.$, Fig. 1e), and reduced sucrose intake in the SPT $\left(F_{5,36}=3.620, p<0.01\right.$, Fig. 1f $)$. The robustness of these effects varied across the different Ris dose groups. Compared with the CRS + Vehicle group, the CRS + Ris groups had significantly decreased immobility times in the TST $\left(F_{5,36}=6.736, p<0.001\right.$ for all four Ris dose groups, Fig. 1e) and FST $\left(F_{5,36}=6.135, p<0.05\right.$ for $0.6 \mathrm{mg} / \mathrm{kg}$ Ris group, $p<0.001$ for $1.0 \mathrm{mg} / \mathrm{kg}$ Ris group, Fig. 1d), as well as marked increases in total distance traveled in the OFT $\left(F_{5,36}=12.309, p<0.05\right.$ for $0.1 \mathrm{mg} / \mathrm{kg}$ Ris, $p<0.01$ for $0.3 \mathrm{mg} / \mathrm{kg}$ Ris, $p<0.001$ for $0.6 \mathrm{mg} / \mathrm{kg}$ Ris, $p<$ 0.0001 for $1 \mathrm{mg} / \mathrm{kg}$ Ris, Fig. 1b). The latter two aforementioned behavioral datasets show Ris dose dependency. Ris did not affect the other tested behaviors significantly in CRS mice at any of the four doses tested.
Systemic Ris downregulated GluN2B and BDNF expression and inhibited neuronal activity in the $\mathrm{LHb}$

Expression of GluN2B, NGF, BDNF, NT4, NT3, and c-Fos proteins in the $\mathrm{LHb}$ was detected after administration of Ris $(1 \mathrm{mg} / \mathrm{kg}$, i.p.) in CRS mice. Compared with the Con + Vehicle group, the CRS + Vehicle group had greater levels of GluN2B $\left(F_{2,6}=20.485\right)$, BDNF $\left(F_{2,6}=29.946\right.$, and $c$-Fos $\left(F_{2,6}=56.653\right)$ expression; meanwhile, compared with the CRS + Vehicle group, the CRS + Ris group had lower levels of GluN2B $\left(F_{2,6}=20.485\right)$, BDNF $\left(F_{2,6}=29.946\right)$, and cFos $\left(F_{2,6}=56.653\right)$ expression (all $p<0.01$, Fig. $2 c$, e). Immunohistochemistry showed that CRS increased numbers of c-Fos positive neurons in the $\operatorname{LHb}\left(F_{2,117}=40.876, p<0.001\right.$ vs. Con group, Fig. $2 b, d)$, and that Ris ( $1 \mathrm{mg} / \mathrm{kg}$, i.p.) reduced c-Fos positive neurons in the LHb of CRS mice $\left(F_{2,117}=40.876, p<0.001\right.$ vs. CRS + Vehicle group, Fig. 2b, d). Neither CRS nor Ris altered NGF, NT4, or NT3 protein levels in the LHb significantly.

Intra-LHb injection of Ris improved despair-like behavior Compared with non-CRS surgery-control mice given intra-LHb infusions of only vehicle, CRS mice given intra-LHb infusions of only vehicle showed stable depression-like behaviors, including decreased total distance $\left(F_{2,20}=4.151, p<0.05\right.$, Fig. $\left.3 d\right)$ and central area duration $\left(F_{2,20}=3.625, p<0.05\right.$, Fig. 3e) in the OFT, increased immobility times in the FST $\left(F_{2,19}=10.626, p<0.01\right.$, Fig. 3f) and TST $\left(F_{2,19}=8.630, p<0.05\right.$, Fig. $\left.3 g\right)$, and reduced sucrose intake in the SPT $\left(F_{2,20}=14.459, p<0.001\right.$, Fig. $\left.3 h\right)$. Compared with surgery-control CRS mice given intra-LHb injections of only vehicle, CRS mice given intra-LHb injections of Ris ( $4 \mathrm{nmol}$ in $0.2 \mu \mathrm{l} / \mathrm{side}$ ) had significantly decreased immobility times 
A

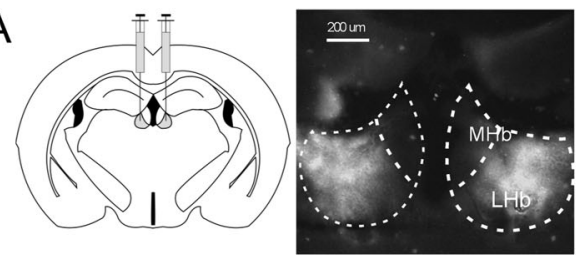

D

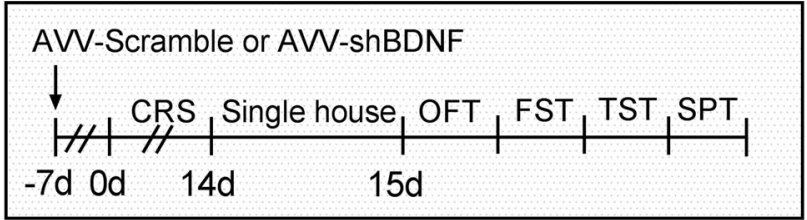

B

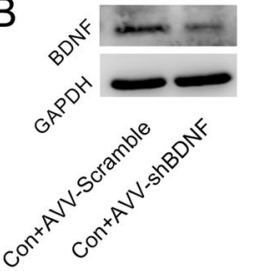

C

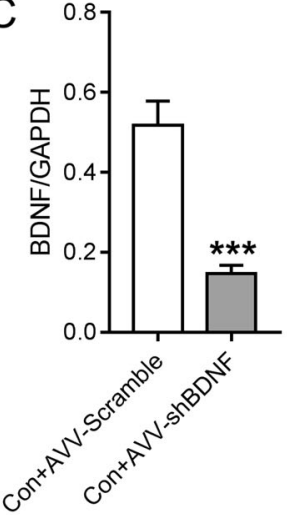

E

OFT

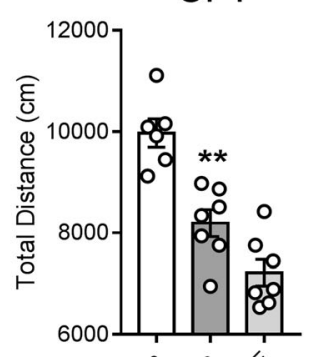

F OFT

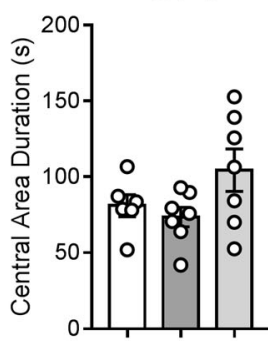

G

$\mathrm{H}$

TST
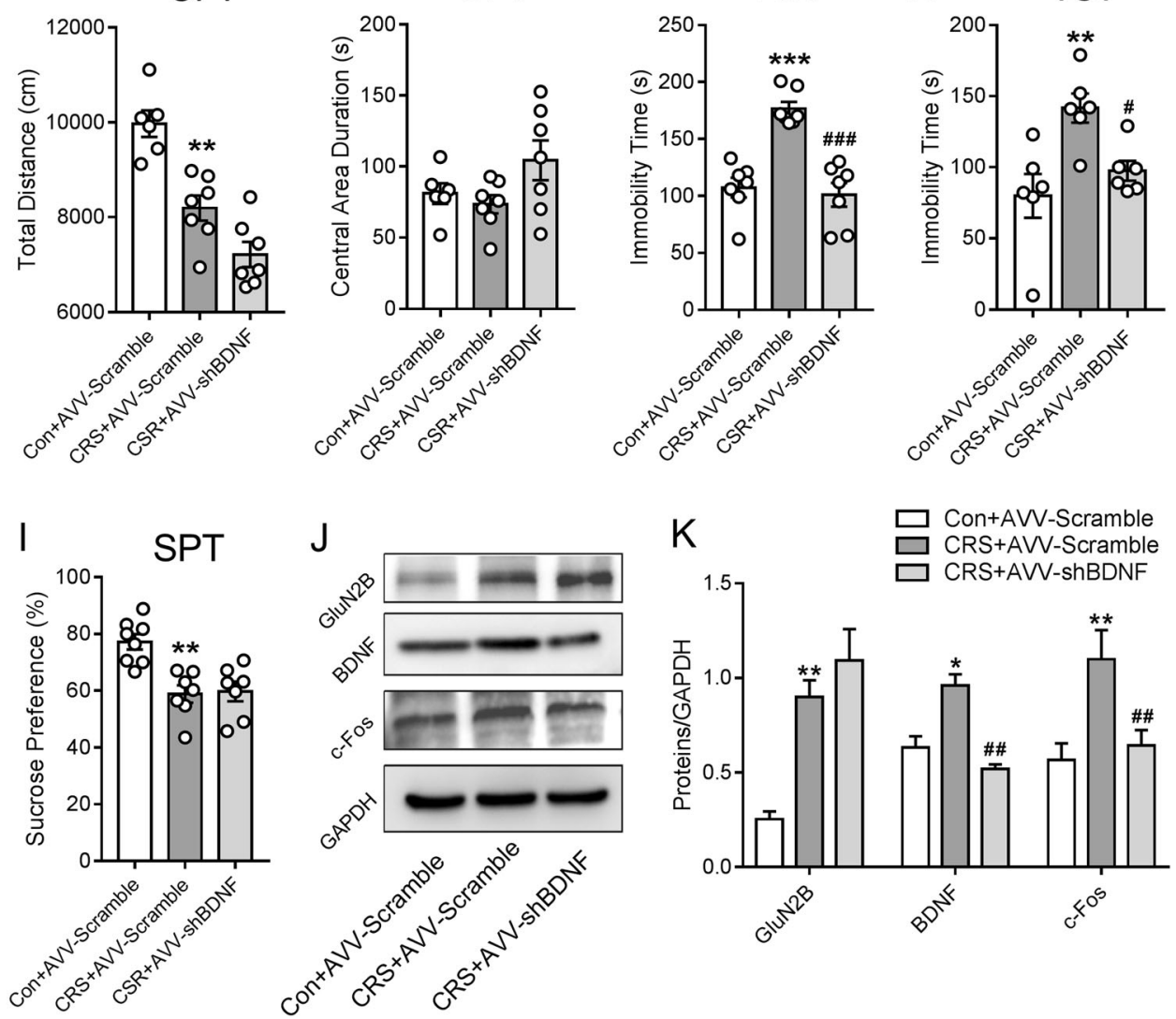

Fig. 4 Knockdown of BDNF in the LHb prevents the development of CRS-induced depressive-like behavior and normalizes expression of BDNF and c-Fos. a Map of AVV injection (left) and a coronal section showing the site of AVV injection in the LHb (right; green, enhanced green fluorescence protein). b Representative western blot showing the interference efficiency of AAV-shBDNF. c Quantitative analysis of BDNF expression in the LHb from the experiment shown in panel B $(n=4-6 /$ group). d Timeline of AVV injection and behavioral testing. e Total distance in the OFT, $n=6-7 /$ group. $\mathbf{f}$ Central area duration in the OFT, $n=6-7 /$ group. $\mathbf{g}$ Immobility time in the FST, $n=7 / g r o u p . \mathbf{h}$ Immobility time in the TST, $n=6 /$ group. i Sucrose preference in the SPT, $n=7-8 /$ group. $\mathbf{j}$ Representative western blot protein bands shown the expression of GluN2B, BDNF, and c-Fos protein levels. k Quantitative analysis of protein expression from the experiment shown in (j) $(n=4-6 /$ group). Data are means \pm SEMs; ${ }^{*} p<0.05 ;{ }^{* *} p<0.01 ;{ }^{* * *} p<0.001$ vs. Con + AAV-Scramble group; ${ }^{\#} p<0.05 ;{ }^{\# \#} p<0.01 ;{ }^{\# \# \#} p<0.001$ vs. CRS + AAV-Scramble group.

in the FST $\left(F_{2,19}=10.626, p<0.01\right.$, Fig. $\left.3 f\right)$ and TST $\left(F_{2,19}=8.630, p\right.$ $<0.01$, Fig. 3g). The behavior of non-CRS control mice was not affected significantly by local injection of Ris ( $4 \mathrm{nmol}$ in $0.2 \mu \mathrm{l} / \mathrm{side}$ ) into the LHb (Fig. S1).

BDNF knockdown in the LHb prevented CRS-induced despair-like behavior and inhibited neuronal activity in the LHb

To further test the role of BDNF in the LHb in the development of depression-like behaviors in mice, we tested the effects of CRS on depressive-like behaviors after specific knockdown BDNF in the $\mathrm{LHb}$. The interference efficiency of AVV-shBDNF was confirmed in western blot assays. Compared with the Con + AVV-Scramble group, the Con + AVV-shBDNF group had decreased BDNF levels 3 weeks after intra-LHb infusion of AAV-shBDNF $(t=12.328, p<$ $0.001 \mathrm{t}$ test, Fig. $4 \mathrm{~b}, \mathrm{c}$ ). Behavioral testing of mice subjected to CRS 1 week after AAV-shBDNF or AAV-Scramble infusion showed that, compared with the Con + AVV-Scramble group, the CRS + AVVScramble group showed greater depressive-like behaviors, 
including decreased total distance $\left(F_{2,17}=2.870, p<0.01\right.$, Fig. $\left.4 \mathrm{e}\right)$ in the OFT, increased immobility times in the FST $\left(F_{2,18}=24.566, p\right.$ $<0.001$, Fig. 4g) and TST $\left(F_{2,15}=7.743, p<0.01\right.$, Fig. 4h), and reduced sucrose intake in the SPT $\left(F_{2,19}=11.733, p<0.01\right.$, Fig. $\left.4 \mathrm{i}\right)$. Interestingly, compared with CRS mice that received AVVScramble, CRS mice given intra-LHb infusions of AAV-shBDNF exhibited attenuated development of CRS-induced despair-like behavior as reflected by decreased immobility times in the FST $\left(F_{2,18}=24.566, p<0.001\right.$, Fig. $\left.4 g\right)$ and TST $\left(F_{2,15}=7.743, p<0.05\right.$, Fig. 4h). The shBDNF treatment did not affect the other depressive-like behaviors examined in CRS mice significantly and had no significant behavioral effects in non-CRS control mice (Fig. S2). Finally, western blots showed that BDNF knockdown with intra-LHb infusion of AAV-shBDNF blocked CRS-induced increases in $\operatorname{BDNF}\left(F_{2,6}=21.287, p<0.01\right)$ and c-Fos $\left(F_{2,6}=26.582, p<0.01\right)$, without affecting GluN2B levels (Fig. 4j, k).

\section{DISCUSSION}

Although Ris, a specific GluN2B receptor antagonist, is being examined in a phase II clinical study, the mechanism mediating its antidepressant effect is unclear. Consistent with previous studies [33], our study showed that systemic injection of Ris improved depression-like behaviors in CRS mice, as reflected by the mice covering more distance in the OFT and spending less time immobile in the FST and TST. In a previous study, systemic administration of Ris was shown to inhibit ${ }_{3} \mathrm{H}$ ] Ro 25-6981 (a specific GluN2B antagonist) binding of GluN2B-containing NMDARs in mouse and rat forebrain [35] and these results suggest that Ris may be able to cross the blood brain barrier. In this study, we found that systemic injection of Ris reduced GluN2B expression in the LHb of CRS mice. Of particular importance, our findings showing that local intra-LHb injection of Ris improved despair-like behavior of CRS mice in the FST and TST suggest that Ris alleviated despair-like behavior in mice, at least in part, through the LHb.

In the current study, Ris both downregulated GluN2B expression and reduced BNDF levels in the LHb. In a recent study using a stress-induced animal model of depression, the LHb was shown to have elevated activation of the transcription factor CREB (cyclic AMP response element binding protein), as reflected by phosphorylated CREB levels, and facilitated long-term synaptic potentiation [36]. CREB binding to the BDNF promoter, and consequent upregulation of BDNF expression, have been shown to be initiated by $\mathrm{Ca}^{2+}$ influx [37]. Thus, Ris may downregulate GluN2B expression and decrease $\mathrm{Ca}^{2+}$ influx, thereby regulating calmodulin-dependent protein kinases and inhibiting expression of BDNF in the LHb.

Although BDNF is the neurotrophin that has been most closely associated with depression $[38,39]$, its role in the pathophysiology and treatment of depression remains controversial. Human patients with MDD have significantly reduced serum levels of BDNF, compared with healthy controls, and antidepressant treatments can normalize serum BDNF levels in these patients [40]. Likewise, antidepressant agents can reverse stressor-induced decreases in BDNF levels in the hippocampus and MPFC in rats $[24,41]$. Specific knockdown of BDNF in the dorsal dentate gyrus of the hippocampus induced despair-like and anhedonia-like behaviors, whereas specific knockdown of BDNF in the ventral subiculum of the hippocampus induced only anhedonia-like behavior [42]. Meanwhile, BNDF levels in the nucleus accumbens (NAc) have been reported to be increased after chronic social defeat stress, and mice with high BDNF levels in the NAc were found to be more susceptible to defeat stress [43]. BDNF variance in the NAc might help to explain why BDNF deletion failed to induce depressive-like behaviors in a prior report [44]. The associations of depression-like behaviors with reduced hippocampal BDNF but elevated NAc BDNF suggest that these two brain regions may have differing roles in the development of depressive symptoms. We speculate that BDNF involvement in MDD pathogenesis encompasses multiple influences from different brain regions.

Our results showed that specific knockdown of BDNF in the LHb prevented the development of despair-like behavior following CRS and decreased c-Fos expression in the LHb. BDNF treatment has been shown to induce expression of c-Fos (a marker of neuronal activity) in rat primary cortical neurons [45], and numerous studies have reported that increased activity of $\mathrm{LHb}$ neurons is an important factor in the development of depression symptoms $[27,46]$. Thus, Ris may improve CRS-induced despair-like behavior by reducing the expression of BDNF proteins in the LHb, thereby inhibiting the activity of $\mathrm{LHb}$ neurons.

$\mathrm{LHb}$ neurons project to midbrain areas (the substantia nigra compacta/ventral tegmental area and the median raphe nucleus/ dorsal raphe nucleus) and control their activities that are involved in the release of dopamine and 5-HT neurotransmitters respectively [47]. The suppressive effect of $\mathrm{LHb}$ activation on these brain regions is mainly mediated by GABAergic neurons in rostromedial tegmental nucleus (RMTg) $[48,49]$. Inhibition of $\mathrm{LHb}$ afferents to the downstream RMTg has been shown to reduce immobility time in the FST, without having a significant effect on sucrose intake in the SPT [50], which is consistent with our results in behavioral tests. Interestingly, genetic deletion of GluN2B in the $\mathrm{mPFC}$ results in enhanced excitatory synaptic drive onto the $\mathrm{mPFC}$ from upstream regions in the medial dorsal thalamus and reduces despair-like behavior [51]. Thus, it is possible that GluN2B receptor antagonism in the $\mathrm{LHb}$ might alleviate despair-like behavior via effects on incoming afferents or downstream brain regions.

In conclusion, Ris alleviates CRS-induced despair-like behavior in the $\mathrm{LHb}$ of mice and this alleviation may involve reduced BDNF expression, and consequently reduced neuronal activity, in the $\mathrm{LHb}$. It remains to be clarified how Ris affects the activity and molecular expression profiles of different $\mathrm{LHb}$ neuron types. Furthermore, it will be important to explore Ris effects on brain regions that are upstream and downstream of the $\mathrm{LHb}$.

\section{FUNDING AND DISCLOSURE}

This study was supported by the National Natural Science Foundation of China (nos: 91332117 and 31671207) and Program for JLU Science and Technology Innovative Research Team (2017TD-30). The authors declare no competing interests.

\section{ACKNOWLEDGEMENTS}

We thank Xin Liu and Lijiang MA for their technical assistance in this study.

\section{AUTHOR CONTRIBUTIONS}

TL designed the study, conducted animal experiments, and wrote the paper. DD conducted the molecular experiments. MS analyzed the data. YS directed the experimental techniques of virus injection and immunohistochemistry. $\mathrm{XL}$ provided logistic support to the study. $\mathrm{HZ}$ designed the study, supervised all aspects of the study, and wrote the paper.

\section{ADDITIONAL INFORMATION}

Supplementary Information accompanies this paper at (https://doi.org/10.1038/ s41386-020-0652-9).

Publisher's note Springer Nature remains neutral with regard to jurisdictional claims in published maps and institutional affiliations.

\section{REFERENCES}

1. World Organization Health. Depression. 2018. https://www.who.int/news-room/ fact-sheets/detail/depression. Accessed 4 Dec 2019.

2. Caraci F, Calabrese F, Molteni R, Bartova L, Dold M, Leggio GM, et al. International Union of Basic and Clinical Pharmacology CIV: the neurobiology of treatment- 
resistant depression: from antidepressant classifications to novel pharmacological targets. Pharmacol Rev. 2018;70:475-504.

3. Sanacora G, Treccani G, Popoli M. Towards a glutamate hypothesis of depression: an emerging frontier of neuropsychopharmacology for mood disorders. Neuropharmacology. 2012;62:63-77.

4. Jaso BA, Niciu MJ, ladarola ND, Lally N, Richards EM, Park M, et al. Therapeutic modulation of glutamate receptors in major depressive disorder. Curr Neuropharmacol. 2017;15:57-70.

5. Kucukibrahimoglu E, Saygin MZ, Caliskan M, Kaplan OK, Unsal C, Goren MZ. The change in plasma GABA, glutamine and glutamate levels in fluoxetine- or Scitalopram-treated female patients with major depression. Eur J Clin Pharmacol. 2009;65:571-7.

6. Fogaca MV, Fukumoto K, Franklin T, Liu RJ, Duman CH, Vitolo OV, et al. N-MethylD-aspartate receptor antagonist $d$-methadone produces rapid, mTORC1dependent antidepressant effects. Neuropsychopharmacology. 2019;44:2230-8.

7. Ghasemi M, Phillips C, Fahimi A, McNerney MW, Salehi A. Mechanisms of action and clinical efficacy of NMDA receptor modulators in mood disorders. Neurosci Biobehav Rev. 2017;80:555-72.

8. Ghasemi M, Phillips C, Trillo L, De Miguel Z, Das D, Salehi A. The role of NMDA receptors in the pathophysiology and treatment of mood disorders. Neurosci Biobehav Rev. 2014;47:336-58.

9. Yilmaz A, Schulz D, Aksoy A, Canbeyli R. Prolonged effect of an anesthetic dose of ketamine on behavioral despair. Pharmacol Biochem Behav. 2002;71:341-4.

10. Berman RM, Cappiello A, Anand A, Oren DA, Heninger GR, Charney DS, et al. Antidepressant effects of ketamine in depressed patients. Biol Psychiatry. 2000;47:351-4.

11. Krystal JH, Karper LP, Seibyl JP, Freeman GK, Delaney R, Bremner JD, et al. Subanesthetic effects of the noncompetitive NMDA antagonist, ketamine, in humans. Psychotomimetic, perceptual, cognitive, and neuroendocrine responses. Arch Gen Psychiatry. 1994;51:199-214.

12. Regan $\mathrm{MC}$, Romero-Hernandez $\mathrm{A}$, Furukawa $\mathrm{H}$. A structural biology perspective on NMDA receptor pharmacology and function. Curr Opin Struct Biol. 2015;33:68-75.

13. Schorge S, Colquhoun D. Studies of NMDA receptor function and stoichiometry with truncated and tandem subunits. J Neurosci. 2003;23:1151-8.

14. Lang E, Mallien AS, Vasilescu AN, Hefter D, Luoni A, Riva MA, et al. Molecular and cellular dissection of NMDA receptor subtypes as antidepressant targets. Neurosci Biobehav Rev. 2018;84:352-8.

15. Miller $\mathrm{OH}$, Yang L, Wang CC, Hargroder EA, Zhang $Y$, Delpire $E$, et al. GluN2Bcontaining NMDA receptors regulate depression-like behavior and are critical for the rapid antidepressant actions of ketamine. Elife. 2014;3:e03581.

16. Kiselycznyk C, Jury NJ, Halladay LR, Nakazawa K, Mishina M, Sprengel R, et al. NMDA receptor subunits and associated signaling molecules mediating antidepressant-related effects of NMDA-GluN2B antagonism. Behav Brain Res. 2015;287:89-95.

17. Ibrahim L, Diaz Granados N, Jolkovsky L, Brutsche N, Luckenbaugh DA, Herring WJ, et al. A Randomized, placebo-controlled, crossover pilot trial of the oral selective NR2B antagonist MK-0657 in patients with treatment-resistant major depressive disorder. J Clin Psychopharmacol. 2012;32:551-7.

18. Preskorn SH, Baker B, Kolluri S, Menniti FS, Krams M, Landen JW. An innovative design to establish proof of concept of the antidepressant effects of the NR2B subunit selective $\mathrm{N}$-methyl-D-aspartate antagonist, CP-101,606, in patients with treatment-refractory major depressive disorder. J Clin Psychopharmacol. 2008;28:631-7

19. Lima-Ojeda JM, Vogt MA, Pfeiffer N, Dormann C, Kohr G, Sprengel R, et al. Pharmacological blockade of GluN2B-containing NMDA receptors induces antidepressant-like effects lacking psychotomimetic action and neurotoxicity in the perinatal and adult rodent brain. Prog Neuropsychopharmacol Biol Psychiatry. 2013;45:28-33.

20. Wang CC, Held RG, Chang SC, Yang L, Delpire E, Ghosh A, et al. A critical role for GluN2B-containing NMDA receptors in cortical development and function. Neuron. 2011;72:789-805.

21. deBacker J, Hawken ER, Normandeau CP, Jones AA, Di Prospero C, Mechefske E, et al. GluN2B-containing NMDA receptors blockade rescues bidirectional synaptic plasticity in the bed nucleus of the stria terminalis of cocaine self-administering rats. Neuropsychopharmacology. 2015;40:394-405.

22. Chao MV. Neurotrophins and their receptors: a convergence point for many signalling pathways. Nat Rev Neurosci. 2003;4:299-309.

23. Huang EJ, Reichardt LF. Neurotrophins: roles in neuronal development and function. Annu Rev Neurosci. 2001:24:677-736.

24. Li SX, Han Y, Xu LZ, Yuan K, Zhang RX, Sun CY, et al. Uncoupling DAPK1 from NMDA receptor GluN2B subunit exerts rapid antidepressant-like effects. Mol Psychiatry. 2018;23:597-608.

25. Castonguay D, Dufort-Gervais J, Menard C, Chatterjee M, Quirion R, Bontempi B, et al. The tyrosine phosphatase STEP is involved in age-related memory decline. Curr Biol. 2018;28:1079-89.e4.
26. Khan AM, Stanley BG, Bozzetti L, Chin C, Stivers C, Curras-Collazo MC. N-methyl-Daspartate receptor subunit NR2B is widely expressed throughout the rat diencephalon: an immunohistochemical study. J Comp Neurol. 2000;428:428-49.

27. Yang $\mathrm{Y}$, Wang $\mathrm{H}, \mathrm{Hu} \mathrm{J}, \mathrm{Hu} \mathrm{H}$. Lateral habenula in the pathophysiology of depression. Curr Opin Neurobiol. 2018;48:90-6.

28. Caldecott-Hazard S, Mazziotta J, Phelps M. Cerebral correlates of depressed behavior in rats, visualized using 14C-2-deoxyglucose autoradiography. J Neurosci. 1988;8:1951-61.

29. Yang LM, Hu B, Xia YH, Zhang BL, Zhao H. Lateral habenula lesions improve the behavioral response in depressed rats via increasing the serotonin level in dorsal raphe nucleus. Behav Brain Res. 2008;188:84-90.

30. Yang Y, Cui Y, Sang K, Dong Y, Ni Z, Ma S, et al. Ketamine blocks bursting in the lateral habenula to rapidly relieve depression. Nature. 2018;554:317-22.

31. Schneider TM, Beynon C, Sartorius A, Unterberg AW, Kiening KL. Deep brain stimulation of the lateral habenular complex in treatment-resistant depression: traps and pitfalls of trajectory choice. Neurosurgery. 2013;72:ons184-93.

32. Kim KS, Han PL. Optimization of chronic stress paradigms using anxiety- and depression-like behavioral parameters. J Neurosci Res. 2006;83:497-507.

33. Garner R, Gopalakrishnan S, McCauley JA, Bednar RA, Gaul SL, Mosser SD, et al. Preclinical pharmacology and pharmacokinetics of CERC-301, a GluN2B-selective N-methyl-D-aspartate receptor antagonist. Pharmacol Res Perspect. 2015;3: e00198.

34. Powell TR, Fernandes C, Schalkwyk LC. Depression-related behavioral tests. Curr Protoc Mouse Biol. 2012;2:119-27.

35. Fernandes A, Wojcik T, Baireddy P, Pieschl R, Newton A, Tian Y, et al. Inhibition of in vivo [(3)H]MK-801 binding by NMDA receptor open channel blockers and GluN2B antagonists in rats and mice. Eur J Pharmacol. 2015;766:1-8.

36. Park H, Rhee J, Park K, Han JS, Malinow R, Chung C. Exposure to stressors facilitates long-term synaptic potentiation in the lateral habenula. J Neurosci. 2017;37:6021-30.

37. Tao X, Finkbeiner S, Arnold DB, Shaywitz AJ, Greenberg ME. Ca2+ influx regulates BDNF transcription by a CREB family transcription factor-dependent mechanism. Neuron. 1998;20:709-26.

38. Castren E, Rantamaki T. The role of BDNF and its receptors in depression and antidepressant drug action: reactivation of developmental plasticity. Dev Neurobiol. 2010;70:289-97.

39. Bjorkholm C, Monteggia LM. BDNF-a key transducer of antidepressant effects. Neuropharmacology. 2016;102:72-9.

40. Duman RS, Monteggia LM. A neurotrophic model for stress-related mood disorders. Biol Psychiatry. 2006;59:1116-27.

41. Neto FL, Borges G, Torres-Sanchez S, Mico JA, Berrocoso E. Neurotrophins role in depression neurobiology: a review of basic and clinical evidence. Curr Neuropharmacol. 2011;9:530-52.

42. Taliaz D, Stall N, Dar DE, Zangen A. Knockdown of brain-derived neurotrophic factor in specific brain sites precipitates behaviors associated with depression and reduces neurogenesis. Mol Psychiatry. 2010;15:80-92.

43. Krishnan V, Han MH, Graham DL, Berton O, Renthal W, Russo SJ, et al. Molecular adaptations underlying susceptibility and resistance to social defeat in brain reward regions. Cell. 2007;131:391-404.

44. Duman RS, Voleti B. Signaling pathways underlying the pathophysiology and treatment of depression: novel mechanisms for rapid-acting agents. Trends Neurosci. 2012;35:47-56

45. Kuzniewska B, Rejmak E, Malik AR, Jaworski J, Kaczmarek L, Kalita K. Brain-derived neurotrophic factor induces matrix metalloproteinase 9 expression in neurons via the serum response factor/c-Fos pathway. Mol Cell Biol. 2013;33: 2149-62.

46. Li B, Piriz J, Mirrione M, Chung C, Proulx CD, Schulz D, et al. Synaptic potentiation onto habenula neurons in the learned helplessness model of depression. Nature. 2011;470:535-9.

47. Herkenham M, Nauta WJ. Efferent connections of the habenular nuclei in the rat. J Comp Neurol. 1979;187:19-47.

48. Sego C, Goncalves L, Lima L, Furigo IC, Donato J Jr., Metzger M. Lateral habenula and the rostromedial tegmental nucleus innervate neurochemically distinct subdivisions of the dorsal raphe nucleus in the rat. J Comp Neurol. 2014;522:1454-84

49. Brown PL, Palacorolla H, Brady D, Riegger K, Elmer Gl, Shepard PD. Habenulainduced inhibition of midbrain dopamine neurons is diminished by lesions of the rostromedial tegmental nucleus. J Neurosci. 2017;37:217-25.

50. Proulx CD, Aronson S, Milivojevic D, Molina C, Loi A, Monk B, et al. A neural pathway controlling motivation to exert effort. Proc Natl Acad Sci USA. 2018;115:5792-7.

51. Miller $\mathrm{OH}$, Bruns A, Ben Ammar I, Mueggler T, Hall BJ. Synaptic regulation of a thalamocortical circuit controls depression-related behavior. Cell Rep. 2017;20:1867-80. 